\title{
A diet rich in fruit and whole grains is associated with a low risk of type 2 diabetes mellitus: findings from a case-control study in South China
}

\author{
Yanbin $\mathrm{Ye}^{1, *}\left(\mathbb{0}\right.$, Shuyu Zhuo ${ }^{1}$, Wei Lu ${ }^{1}$, Kaiyin $\mathrm{He}^{2}, \mathrm{Yi} \mathrm{Sui}^{1}$, Yanbing $\mathrm{Li}^{3}$, \\ Yuming $\mathrm{Chen}^{4, *}$ (1), Shangling Wu ${ }^{1}$, Peiyan Chen ${ }^{1}$ and Shi Fang ${ }^{1}$ \\ 'Department of Clinical Nutrition, The First Affiliated Hospital of Sun Yat-sen University, 58 \# Zhongshan Road 2, \\ Guangzhou 510080, Guangzhou, China: ${ }^{2}$ Department of Gastroenterology, The First Affiliated Hospital of Jinan \\ University, Guangzhou, Guangzhou, China: ${ }^{3}$ Department of Endocrinology, The First Affiliated Hospital of Sun Yat-sen \\ University, Guangzhou, Guangzhou, China: ${ }^{4}$ Department of Medical Statistics and Epidemiology, School of Public \\ Health, Sun Yat-sen University, 74\# Zhongshan Road 2, Guangzhou 510089, Guangzhou, China
}

Submitted 20 May 2020: Final revision received 14 November 2020: Accepted 30 November 2020: First published online 15 December 2020

\begin{abstract}
Objective: Various foods are associated with or protect against type 2 diabetes mellitus (T2DM). This study was to examine the associations of foods and food patterns with the risk of T2DM in South China.

Design: Case-control study.

Setting: The dietary patterns were identified by a principal components factor analysis. Univariable and multivariable conditional logistic regression analyses were used to analyse the associations between food groups and dietary patterns and the risk of T2DM.

Participants: A total of 384 patients with T2DM and 768 controls.

Results: After adjustment for total energy intake, the standard intake of grains $(228.3 \pm 71.9 v .238 .8 \pm 73.1 \mathrm{~g} / \mathrm{d}, P=0.025)$ and fruits $(109 \pm 90 v .145 \pm 108 \mathrm{~g} / \mathrm{d}$, $P<0.001)$ were lower in T2DM than in controls. Four dietary patterns were identified: (1) high light-coloured vegetables and low grains, (2) high fruits, (3) high red meat and low grains and (4) high dark-coloured vegetable. After adjustment for covariables, multivariable conditional logistic regression analyses showed significant dose-dependent inverse associations between total fruit intake, whole grains intake and the score of the high-fruit dietary pattern (all $P_{\text {for trend }}<0.001$ ) and the risk of T2DM. The adjusted OR (95\% CI) for T2DM comparing the extreme quartiles were $0.46(0.29,0.76)$ for total fruits, $0.48(0.31,0.77)$ for whole grains and 0.42 $(0 \cdot 26,0 \cdot 68)$ for the high-fruit dietary pattern, respectively. Similar associations were observed for all subgroups of fruits (dark-colour and light-colour).

Conclusion: In South China, a diet rich in fruit and whole grains is associated with lower risk of T2DM.
\end{abstract}

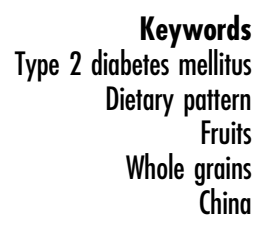

Type 2 diabetes mellitus (T2DM) is a common endocrine disorder characterised by variable degrees of insulin resistance and deficiency, resulting in hyperglycaemia ${ }^{(1)}$. Potential complications of T2DM include CVD, neuropathy, nephropathy, retinopathy and mortality ${ }^{(1)}$. The worldwide prevalence of T2DM was $9 \%$ in men and $7.9 \%$ in women in $2014^{(1)}$. In China, the prevalence of T2DM increased from $2.5 \%$ in $1994^{(2)}$ to $10.9 \%$ in 2013 , with a prevalence of pre-diabetes of $35.7 \%{ }^{(3)}$. Medical expenses related to T2DM also exploded in
China, from USD 0.25 billion in 1993 to USD 8.65 billion in $2009^{(4)}$.

The risk factors for T2DM include obesity, metabolic syndrome, poor diet and lack of exercise ${ }^{(5)}$. Especially, the westernisation of the diet in China over recent decades has been associated with the prevalence of $\mathrm{T}_{2} \mathrm{DM}^{(6)}$. A number of specific foods have been associated with T2DM, including processed meat ${ }^{(7)}$, red meat ${ }^{(8)}$, fried food $^{(9)}$, white rice ${ }^{(10)}$ and sugar-sweetened beverages ${ }^{(11)}$, while higher consumption of cereal fibres ${ }^{(12)}$, fish ${ }^{(13)}$, 
milk $^{(14)}$ and soyabeans (and their isoflavones) ${ }^{(15)}$ are associated with the prevention of T2DM and its complications. Regarding fruits and vegetables, their association with T2DM might be only significant for the lowest quintile ${ }^{(16)}$.

Nevertheless, the studies about the association between dietary patterns and T2DM often report variable and even conflicting results. Indeed, various tools for dietary assessment are available, each with their pros, cons and biases, and they yield variable results ${ }^{(17)}$. Dietary habits are highly cultural, and those cultural dietary patterns are complex and specific ${ }^{(18)}$. T2DM is a complex trait, influenced both by the environment and genetics ${ }^{(19)}$. Since the genetic patterns vary widely across the world, different populations will have a different genetic susceptibility to $\mathrm{T}_{2} \mathrm{DM}^{(20)}$. Therefore, the results of the available studies cannot be generalised to other populations, and studies specific to each population must be conducted in order to improve the understanding of the dietary risk of T2DM in specific populations. A meta-analysis of 16 cohorts showed that adhering to the Mediterranean diet, Dietary Approach to Stop Hypertension (DASH), or Alternative Healthy Eating Index (AHEI) was associated with a lower risk of T2DM, while dietary patterns characterised by meat (red and processed), refined grains, high-fat dairy products, eggs and fried foods were associated with a higher risk of $\mathrm{T} 2 \mathrm{DM}^{(21)}$.

Therefore, the aim of the present study is to determine whether particular foods and dietary patterns have associations with T2DM risk in South China. The results could help guide the residents' daily diet to prevent T2DM.

\section{Methods}

\section{Study design and subjects}

This was a case-control study of permanent adult residents (30-80 years of age) who had been living in Guangzhou (South China) for at least 5 years. This study was conducted according to the guidelines laid down in the Declaration of Helsinki and all procedures involving human subjects were approved by the ICE for Clinical Research and Animal Trials of the First Affiliated Hospital of Sun Yat-sen University. Written informed consent was obtained from all subjects.

From May 2011 to February 2012, most patients with new-onset T2DM at the endocrinology clinic and inpatient ward of the First and Second Affiliated Hospitals of Sun Yat-sen University were recruited. T2DM was confirmed based on their glucose examination reports. Control subjects were recruited using a questionnaire survey and fasting blood glucose (FBG) screening at Nonglin Street Community, Yuexiu District, Guangzhou, and from the otolaryngology department of the First and Second Affiliated Hospitals of Sun Yat-sen University.

The patients with T2DM met the diagnostic criteria of T2DM from the American Diabetes Association in $2007^{(22)}$ and were with new-onset T2DM, that is, $\leq 3$ months since diagnosis. The control group included adults without abnormal glucose metabolism (FBG $<5.6 \mathrm{mmol} / \mathrm{l}$ and 2 -h postprandial blood glucose $<7.8 \mathrm{mmol} / \mathrm{l}$ ). The casecontrol ratio was $1: 2$, and the controls were matched with the T2DM group for sex and age (within \pm 3 years).

The exclusion criteria were (1) severe heart, lung, liver or renal dysfunction, or severe gastrointestinal diseases; (2) thyroid or other endocrine dysfunction; (3) taking hormones; (4) paralysis or walking difficulties; (5) mental disorders or (6) vegetarians. Individuals with FBG of 5.6-7.0 $\mathrm{mmol} / 1$ were excluded.

\section{Data collection and dietary assessment}

A structured questionnaire that included sociodemographic characteristics, lifestyle habits, history of chronic diseases, medical history and dietary habits was completed by all subjects during a face-to-face interview by trained interviewers.

A validated dietary FFQ was used for dietary intake assessment ${ }^{(23)}$. It has seven categories of food, including 79 food items. The participants were asked to report the information on the frequency of each food item intake and its portion size in the last year before diagnosis (patients) or interview (control subjects). Food photographs of portion size were used to help estimate the amount of food consumption. According to the frequency of intake and portion size, the average consumption amount of each food item (g/d) was calculated. For seasonal foods, participants were asked to report on how many months of the year they consumed each item. The average daily intake of each food group and its nutrients was calculated according to the China Food Composition $2002^{(24)}$. The participants were excluded if their FFQ was invalid, that is, more than five food items were missing or if the FFQ resulted in implausible energy intakes ( $<800$ or $>4000 \mathrm{kcal}$ per day). To reduce the complexity of the food data, the 79 food items were aggregated into 30 groups according to similarities in nutrients or processing methods to identify the dietary patterns (see online supplementary material, Supplemental Table S4). We used a questionnaire to estimate the usual physical activity, which including hours and minutes spent on daily activities and leisure activities in the recent 1 year. Total energy expenditure was evaluated according to metabolic equivalents ${ }^{(25)}$.

Physical examinations (height, weight, waist and hip circumferences and blood pressure) and laboratory examinations (FBG for the controls) were examined on the interview morning.

\section{Statistical analysis}

All data of the FFQ were managed using Epidata 3.0 software and exported to SPSS 16.0 (IBM) for statistical analyses. Two-sided $t$ tests were used, and $P$-values $<0.05$ were considered significant. To achieve an approximately normal distribution for statistical analysis, logarithmic transformation was applied to daily energy intake, whereas the square root transformation was applied to other dietary data. 
To evaluate the sociodemographic characteristics, other potential risk factors, and food groups and nutrients, we used independent-samples $t$ test (continuous variables) and chi-square test (categorical variables) for comparison between the case and control groups. All nutrients and food groups were adjusted by the residual energy method ${ }^{(26)}$. The principal component analysis was used for the construction of dietary patterns. The standardised intake values of the 30 food groups (see online supplementary material, Supplemental Tables S4 and S5) were included in the factor analysis. Four factors were retained based on an inspection of the plots. Varimax-rotated by orthogonal transformation was used to obtain the factor loading value of each factor. We calculated the factor scores for each retained factor by the summation of the intakes of each food group and weight by their factor loadings (a factor loading $\geq 0.1$ showed the main component of the factor). For the analysis of the associations between dietary factors and T2DM, the intake of each food, nutrient and score of each dietary pattern was divided into quartiles (Q1-Q4, Q1 as the reference group) based on the distribution of dietary intake among the control group and then applied the gender-specific cut-offs to the case. The Cox conditional logistic regression was conducted with the bottom quartile group (Q1) as the reference group. Univariable and multivariable analyses were used to calculate the OR with the corresponding $95 \%$ CI. The univariable analyses were not adjusted, while in multivariable analyses, 13 confounding factors were adjusted for (age, sex, marital status, educational level, occupation, smoking, alcohol consumption, tea drinking, economic conditions energy intake, physical activity, family history of T2DM and fibre intake (for dietary pattern only)). The stepwise method was used for including the covariables. The ordinal values for dietary intake and pattern categories were also included as continuous variables in the linear trend tests. The associations between the subcategories of grains, fruits and vegetables and the risk of T2DM were also evaluated in this study.

\section{Results}

\section{Recruitment}

In this study, 600 diabetic patients (30-80 years of age) were recruited at the First and Second Affiliated Hospitals of Sun Yat-sen University and Mapenggang Community, Nonglin Street, Yuexiu District. After initial screening and rescreening, 384 eligible patients were enrolled. For the control group, 630 people from the community and 220 patients in other departments of the hospitals were recruited, matched for age and sex with those of the T2DM group. According to the inclusion/exclusion criteria, 768 subjects were included in the control group (578 from the community and 190 from the hospitals). The recruitment process and details are shown in Fig. 1.

\section{Characteristics of the subjects}

Table 1 shows the sociodemographic characteristics of the subjects. Over $2 / 3$ (66.6\% of the T2DM group and $68.0 \%$ of the control group) of the subjects were 50-70 years of age. The frequency of T2DM was higher among subjects $<50$ years of age. Compared with the control subjects, the subjects with T2DM were more likely to have a higher education level, household income, energy intake, BMI, alcohol consumption and the likelihood of having a family history of $\mathrm{T} 2 \mathrm{DM}(34.4 \% v \cdot 11 \cdot 2 \%)$. The other characteristics of the subjects were not significantly different between the two groups.

\section{Daily average intake of food categories and nutrients}

Compared with controls, patients with T2DM had a lower intake of total grains $(228.3 \pm 71.9$ v. $238.8 \pm 73.1 \mathrm{~g} / \mathrm{d}$, $P=0.025)$ and fruits $(109 \pm 90$ v. $145 \pm 108 \mathrm{~g} / \mathrm{d}$, $P<0.001)$, a higher intake of animal foods and cooking oil $(243 \pm 129 v .229 \pm 95 \mathrm{~g} / \mathrm{d}, P=0 \cdot 047$; and $26 \cdot 1 \pm 12 \cdot 0$ v. $17.8 \pm 11.6 \mathrm{~g} / \mathrm{d}, P<0.001$, respectively), and a similar intake of milk, soybean and derivatives intake, and other food groups (Table 2).

The average energy intake for the T2DM and control groups was $9075 \pm 2675 \mathrm{~kJ} / \mathrm{d}$ and $7965 \pm 2345 \mathrm{~kJ} / \mathrm{d}$, respectively $(P<0.001)$. The average intake of total protein and fat in the T2DM group was higher than those of the control group $(92.5 \pm 33.1 \quad v . \quad 82.7 \pm 28.2 \mathrm{~g} / \mathrm{d}, \quad 78.6 \pm 19.9 \quad v$. $57.2 \pm 16.7 \mathrm{~g} / \mathrm{d}$, both $P<0.001)$, and the intake of animal protein was higher in the diabetic patients $(51.9 \pm 28.0 \mathrm{v}$. $44 \cdot 2 \pm 22 \cdot 1, P<0 \cdot 001$ ) (Table 2).

\section{Association between six main food categories and risk of type 2 diabetes mellitus}

Table 3 shows the associations between the six food categories and the risk of T2DM. The intake of total grains in women was lower than that of men, while the milk intake was lower in men than in women. Univariable and multivariable conditional logistic regression analyses showed significant dose-dependent inverse correlations between the intake of fruits and milk and the risk of T2DM in all subjects, without and with adjustment for confounders $\left(P_{\text {for trend }}<0 \cdot 05-0.01\right)$. After adjustment for age, sex and 11 other potential factors, the significant associations still remained strong in model 2: the OR $(95 \% \mathrm{CI})$ of T2DM for the highest quartile, compared with the lowest quartile, were $0.46(0.29,0.72)$ for fruits and $0.51(0.33,0.81)$ for milk. An inverse association was also seen between an appropriate range of intake of total grains and the risk of T2DM, and the OR $(95 \% \mathrm{CI})$ for the second and the third quartile were $0.64(0.41,0.99)$ and $0.55(0.35,0.84)$ $\left(P_{\text {for trend }}=0.081\right)$. The intake of cooking oil significantly increased the risk of T2DM with a strong dose-dependent effect $\left(\mathrm{P}_{\text {for trend }}<0.001\right)$ in both regression models, and the OR $(95 \% \mathrm{CI})$ of T2DM after adjustment for all confounding 


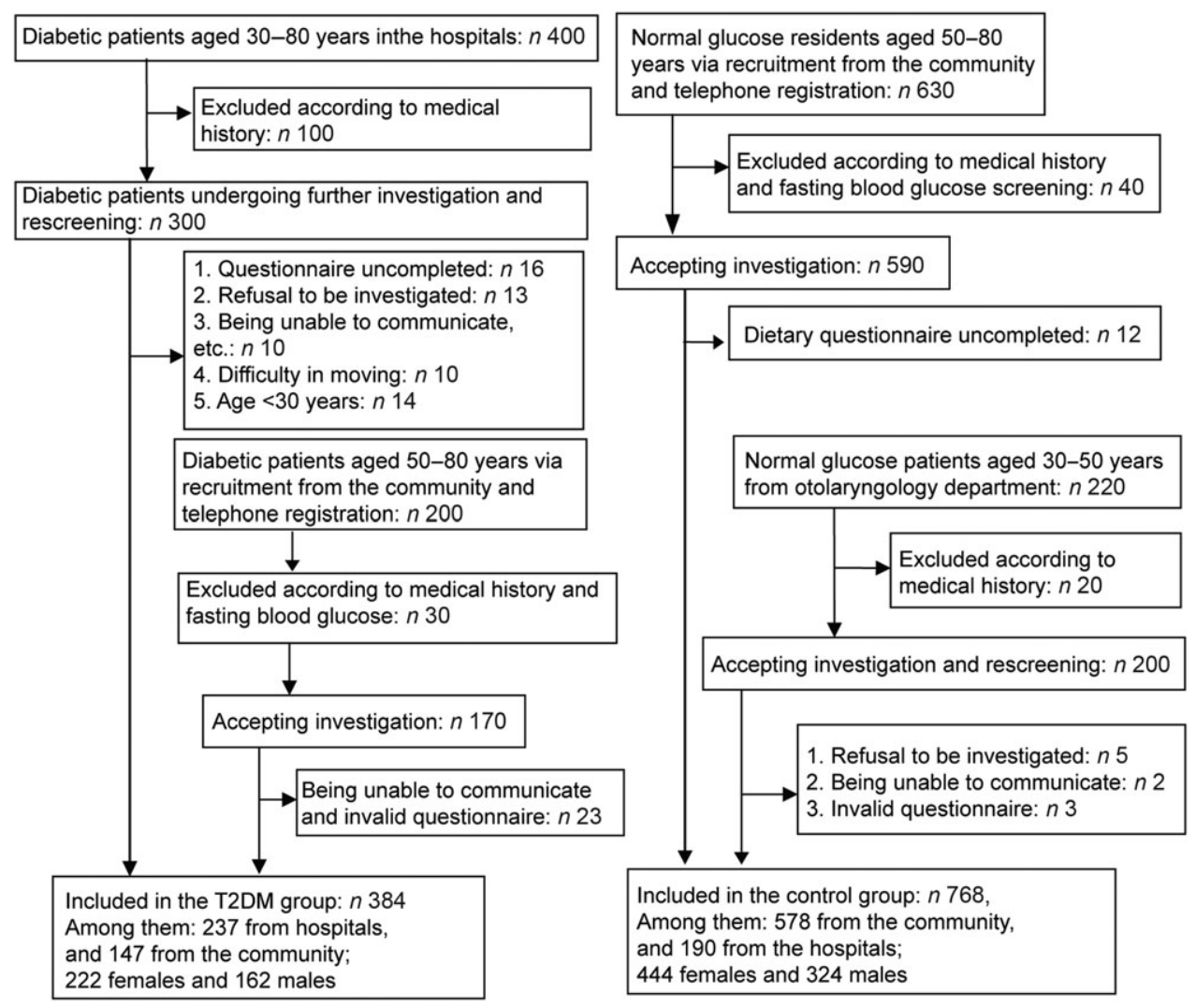

Fig. 1 Patient flowchart. T2DM, type 2 diabetes mellitus

factors for the highest quartile was $10 \cdot 85(6 \cdot 14,19 \cdot 17)$. The intake of vegetables and animal foods did not show any effect on the risk of T2DM in the study population.

\section{Association between grain subcategories and risk of type 2 diabetes mellitus}

The associations between the subgroups of grains and the risk of T2DM were also tested (see online supplementary material, Supplemental Table S1). Total grains were divided into three subgroups: whole grains (with wholewheat bread as the representative member), high-energy grains(Chinese cakes such as radish cakes and water chestnut cakes, cakes, fried snacks and biscuits) and refined grains (rice (including glutinous rice products), porridge, oatmeal, corn flakes, noodles, white bread or steamed bun, white bread or hamburger, barbecued pork buns, and dumplings). The results showed that both univariable and multivariable conditional logistic regression analyses showed a significant dose-dependent inverse correlation between the intake of whole grains and the risk of T2DM ( $\left.P_{\text {for trend }} \leq 0.001\right)$, and the reduction of the risk after adjustment for the confounding factors for the highest quartile $v$. the lowest quartile was $52 \%$ (95\% CI: 23\%, 69\%). There was no association found between high-energy grains and refined grains and the risk of T2DM.

\section{Association between vegetable and fruit subcategories and risk of type 2 diabetes mellitus}

Table 4 and Supplementary Table 2 show the analyses of the associations between vegetable and beans and the risk of T2DM, with the results of subgroup analyses for different kinds of fruit shown in the Supplementary Table S3. Both two logistic regression analyses showed significant dosedependent inverse associations between the intake of lightand dark-coloured fruit subgroups with the risk of T2DM $\left(P_{\text {for trend }}<0.05\right)$, and this inverse association was similar to that observed for total fruits (Tables 2 and 3). After adjustment for the potential confounding factors, the OR (95\% CI) of T2DM for the highest quartile, compared with the lowest quartile, was $0.58(0.37,0.90)$ for dark-coloured fruit and $0.39(025,0.60)$ for light-coloured fruit. The results of analyses of the light- and dark-coloured vegetable subgroups were the same as that for the total vegetables (Table 2 and 3), and their intakes were not correlated with the risk of T2DM. Univariable analyses showed a significant inverse dose-dependent effect of the intake of root vegetables with the risk of T2DM $\left(P_{\text {for trend }}<0 \cdot 01\right)$, but this protective effect disappeared after adjusting for the confounding factors. The significant dose-dependent inverse correlations between the intake of fresh beans and mixed beans and the risk of T2DM were found in univariable conditional logistic regression analyses or multiple adjustment models (models 2, $P_{\text {for trend }}<0.01$ ). The results in Supplementary 
Table 1 Demographic, lifestyle characteristics and selected T2DM risk factors of the study population in Guangzhou, China

\begin{tabular}{|c|c|c|c|c|c|}
\hline \multirow[b]{2}{*}{ Variables } & \multicolumn{2}{|c|}{ Control group } & \multicolumn{2}{|c|}{ T2DM group } & \multirow[b]{2}{*}{$P^{\star}$} \\
\hline & $n$ & $\%$ & $n$ & $\%$ & \\
\hline Sex & & & & & 1.000 \\
\hline Female & 444 & $57 \cdot 8$ & 222 & $57 \cdot 8$ & \\
\hline Male & 324 & $42 \cdot 2$ & 162 & $42 \cdot 2$ & \\
\hline Age & & & & & 1.000 \\
\hline $30-40$ & 102 & $13 \cdot 3$ & 50 & $13 \cdot 1$ & \\
\hline $41-50$ & 117 & $15 \cdot 2$ & 60 & $15 \cdot 6$ & \\
\hline $51-60$ & 307 & $40 \cdot 0$ & 153 & $39 \cdot 8$ & \\
\hline $61-70$ & 215 & $28 \cdot 0$ & 103 & $26 \cdot 8$ & \\
\hline $71-80$ & 27 & 3.5 & 18 & 4.7 & \\
\hline Educational levelt & & & & & $<0.001$ \\
\hline Primary education degree or below & 95 & $12 \cdot 4$ & 81 & $21 \cdot 1$ & \\
\hline Junior high school & 216 & $28 \cdot 1$ & 87 & $22 \cdot 6$ & \\
\hline Senior high school and technical secondary school & 282 & $36 \cdot 7$ & 99 & $25 \cdot 8$ & \\
\hline College degree or above & 175 & $22 \cdot 8$ & 117 & $30 \cdot 5$ & \\
\hline Household income, yuan/month /person $†$ & & & & & $<0.001$ \\
\hline$\leq 500$ yuan & 37 & 4.8 & 13 & 3.4 & \\
\hline $500-1500$ yuan & 254 & $33 \cdot 1$ & 67 & $17 \cdot 4$ & \\
\hline $1501-3000$ yuan & 328 & $42 \cdot 7$ & 144 & $36 \cdot 5$ & \\
\hline$\geq 3001$ yuan & 149 & $19 \cdot 4$ & 160 & $41 \cdot 7$ & \\
\hline Long-term occupation & & & & & 0.051 \\
\hline Light worker & 470 & $61 \cdot 2$ & 242 & 63.0 & \\
\hline Moderate worker & 274 & 335.7 & 120 & $31 \cdot 3$ & \\
\hline Heavy worker & 24 & $3 \cdot 1$ & 22 & 5.7 & \\
\hline Work status in the past year & & & & & 0.484 \\
\hline Full-time & 247 & $32 \cdot 2$ & 137 & $35 \cdot 7$ & \\
\hline Part-time & 29 & 3.8 & 13 & 3.4 & \\
\hline Retired/non-permanent & 492 & 64.0 & 234 & $60 \cdot 9$ & \\
\hline Marital status & & & & & 0.102 \\
\hline Married or cohabiting & 680 & 88.5 & 252 & $92 \cdot 1$ & \\
\hline Widowed, unmarried and divorced & 88 & 11.5 & 32 & 7.9 & \\
\hline Family history ofT2DM & & & & & $<0.001$ \\
\hline Yes & 86 & $11 \cdot 2$ & 132 & $34 \cdot 4$ & \\
\hline No & 682 & $88 \cdot 8$ & 252 & $65 \cdot 6$ & \\
\hline Smoking & & & & & 0.103 \\
\hline Yes & 182 & $23 \cdot 7$ & 108 & $28 \cdot 1$ & \\
\hline No & 586 & $76 \cdot 3$ & 276 & 71.9 & \\
\hline Passive smoking§ & & & & & 0.294 \\
\hline Yes & 235 & $30 \cdot 6$ & 106 & $27 \cdot 6$ & \\
\hline No & 533 & $69 \cdot 4$ & 278 & $72 \cdot 4$ & \\
\hline Alcohol consumptionll & & & & & 0.014 \\
\hline Yes & 73 & 9.5 & 55 & $14 \cdot 3$ & \\
\hline No & 695 & 90.5 & 329 & $82 \cdot 7$ & \\
\hline Tea drinkingी & & & & & 0.196 \\
\hline Yes & 391 & $50 \cdot 9$ & 211 & $55 \cdot 0$ & \\
\hline No & 377 & $49 \cdot 1$ & 173 & $45 \cdot 0$ & \\
\hline Source of subjects & & & & & $<0.001$ \\
\hline Hospital & 190 & 24.7 & 237 & $61 \cdot 7$ & \\
\hline Community & 578 & $75 \cdot 3$ & 147 & $38 \cdot 3$ & \\
\hline Dietary, activity and physical characteristics & & & & & \\
\hline Energy intake (kJ/d) & & & & & $<0.001$ \\
\hline Mean & & & & & \\
\hline SD & & & & & \\
\hline Physical activity(MET-h/d) & & & & & 0.359 \\
\hline Mean & & & & & \\
\hline SD & & & & & \\
\hline $\mathrm{BMI}\left(\mathrm{kg} / \mathrm{m}^{2}\right)$ & & & & & $<0.001$ \\
\hline Mean & & & & & \\
\hline SD & & & & & \\
\hline
\end{tabular}

T2DM, type 2 diabetes mellitus; MET-h/d, metabolic equivalent hours per day.

*The difference in the distribution of categorical variables between the two groups was tested by the chi-square test; the difference in continuous variables was tested using the $t$ test.

†The two independent-samples rank-sum test was used for comparison of the difference in the distribution of education level and family monthly income per capita between the two groups.

$\ddagger$ Smokers referred to those who smoked at least one cigarette per day for more than half a year.

SPassive smokers referred to those living in smoking environments with an average of more than one cigarette or $5 \mathrm{~min} / \mathrm{d}$.

IIDrinkers referred to those who drank at least once a week for six consecutive months.

१Tea drinkers referred to those who had tea at least twice a week. 
Diet and type 2 diabetes mellitus

1497

Table 2 Daily average intake of main food categories and nutrients of the study population in Guangzhou, china

\begin{tabular}{|c|c|c|c|c|c|}
\hline \multirow[b]{2}{*}{ Daily average intake } & \multicolumn{2}{|c|}{ T2DM group ( $n$ 384) } & \multicolumn{2}{|c|}{ Control group ( $n 768)$} & \multirow[b]{2}{*}{$P^{\star}$} \\
\hline & Mean & SD & Mean & SD & \\
\hline \multicolumn{6}{|l|}{ Main food categories } \\
\hline Total intake of grains, $\mathrm{g}$ & $228 \cdot 3$ & 71.9 & $238 \cdot 1$ & $75 \cdot 1$ & 0.025 \\
\hline Total intake of soyabeans and related products, $\mathrm{g}$ & 4.7 & $6 \cdot 3$ & 4.7 & $10 \cdot 4$ & 0.905 \\
\hline Total intake of vegetables, $\mathrm{g}$ & 386 & 282 & 356 & 165 & 0.024 \\
\hline Total intake of fruits, $\mathrm{g}$ & 109 & 90 & 145 & 108 & $<0.001$ \\
\hline Total intake of animal foods, $\mathrm{g}$ & 243 & 129 & 229 & 95 & 0.047 \\
\hline Total intake of eggs, $\mathrm{g}$ & $26 \cdot 9$ & $19 \cdot 6$ & $26 \cdot 4$ & $18 \cdot 6$ & 0.663 \\
\hline Total intake of milk, $\mathrm{g}$ & 126 & 138 & 127 & 142 & 0.924 \\
\hline Total intake of fungi and seaweeds, $g$ & $11 \cdot 8$ & 34.9 & 9.5 & $11 \cdot 3$ & 0.095 \\
\hline Total intake of nuts, $g$ & $13 \cdot 8$ & $26 \cdot 7$ & $16 \cdot 8$ & $26 \cdot 7$ & 0.082 \\
\hline Total intake of cooking oil, $\mathrm{g}$ & $26 \cdot 1$ & 12 & $17 \cdot 8$ & $11 \cdot 6$ & $<0.001$ \\
\hline \multicolumn{6}{|l|}{ Nutrients } \\
\hline Energy, kJ & 9075 & 2675 & 7965 & 2345 & $<0.001$ \\
\hline Carbohydrate, $\mathrm{g}$ & 284.7 & $90 \cdot 9$ & $277 \cdot 3$ & 85.4 & 0.261 \\
\hline Total protein, $\mathrm{g}$ & 92.5 & $33 \cdot 1$ & 82.7 & $28 \cdot 2$ & $<0.001$ \\
\hline Animal protein, $\mathrm{g}$ & 51.9 & 28 & 44.2 & $22 \cdot 1$ & $<0.001$ \\
\hline Vegetable protein, $\mathrm{g}$ & 38.5 & 11.9 & 37.9 & $7 \cdot 8$ & 0.273 \\
\hline Soyabean protein, $\mathrm{g}$ & $2 \cdot 2$ & 2.5 & 2.4 & 2.9 & 0.384 \\
\hline Fat, $g$ & $78 \cdot 6$ & $19 \cdot 9$ & $57 \cdot 2$ & $16 \cdot 7$ & $<0.001$ \\
\hline Dietary fibre, g & $12 \cdot 2$ & 3.5 & 11.4 & $3 \cdot 3$ & $<0.001$ \\
\hline Total isoflavone, $\mathrm{mg}$ & $10 \cdot 3$ & $10 \cdot 6$ & $10 \cdot 6$ & $10 \cdot 7$ & 0.316 \\
\hline Daidzein, mg & 4.3 & 4.4 & $4 \cdot 4$ & 4.5 & 0.408 \\
\hline Genistein, mg & $5 \cdot 8$ & $6 \cdot 1$ & 6.9 & $6 \cdot 0$ & 0.202 \\
\hline Glycitein, mg & 0.56 & 0.67 & 0.6 & 0.8 & 0.812 \\
\hline VitC, mg & $176 \cdot 2$ & 81 & $182 \cdot 6$ & $70 \cdot 8$ & 0.074 \\
\hline Carotene & 5814 & 3030 & 5625 & 2720 & 0.041 \\
\hline $\mathrm{Mg}, \mathrm{mg}$ & $341 \cdot 2$ & $114 \cdot 2$ & 339.3 & 94.2 & 0.001 \\
\hline $\mathrm{Zn}, \mathrm{mg}$ & $17 \cdot \overline{6}$ & 7.5 & $17 \cdot 3$ & $5 \cdot 4$ & $<0.001$ \\
\hline $\mathrm{Se}, \mathrm{mg}$ & $64 \cdot 7$ & $36 \cdot 9$ & $65 \cdot 2$ & 31.9 & 0.007 \\
\hline
\end{tabular}

T2DM, type 2 diabetes mellitus.

${ }^{*}$ Derived from $t$ test.

†Mean daily intake.

$\ddagger$ Standard deviation.

Table S3 indicated null significant difference between different categories of fruits with the risk of T2DM (all $P_{\text {for trend }}>0.05$ ).

\section{Association between dietary patterns score and risk of type 2 diabetes mellitus}

We used the principal component analysis to retain the four main factors associated with T2DM. The details of food composition for 30 food groups and the factor loadings associated with each pattern are given in Supplementary Tables S4 and S5. Each pattern was labelled according to the food groups with high absolute loadings scores. These four factors accounted for 36.94, 13.08, 10.37 and $7.83 \%$ of the variability, respectively, and $68.22 \%$ of the variance in food intake overall. The first factor was a high intake of light-coloured vegetables, root vegetables, nuts, whole milk, and eggs and a low intake of grains, which was named the high-light-coloured vegetables and lowgrain dietary pattern. The second factor was characterised by a high intake of fruits and freshwater fish and a low intake of cooking oil and was named the high-fruit dietary pattern. The third factor was characterised by a high intake of red meat and a low intake of grains and was named the high-meat and low-grain dietary pattern. The fourth factor was characterised by a high intake of dark-coloured vegetables and was named the high-dark-coloured vegetable dietary pattern.

Univariable conditional logistic regression analyses without correction for any factor showed a dosedependent inverse association between the risk of T2DM and the high-fruit pattern $\left(P_{\text {for trend }}<0.001\right)$, but a marginally significant positive association with the high-red meat and low-grain dietary pattern $\left(P_{\text {for trend }}=0.053, \mathrm{Q} 4 v \cdot \mathrm{Q} 1\right.$ : $\mathrm{OR}=1.46,95 \%$ CI 1.02, 2.09) (Table 5). After adjustment for age, sex, marital status, educational level, occupation, smoking, alcohol consumption, tea drinking, economic conditions, energy intake, physical activity, family history of T2DM and dietary fibre, the significant association remained between the high-fruit dietary pattern and the risk of T2DM (model 2 and 3, $P_{\text {for trend }}<0.001$ ), but not for the high-red meat and low-grain dietary pattern ( $P_{\text {for }}$ trend $=0.072$ ). The significant inverse correlation between the high-fruit dietary pattern and the risk of T2DM was observed, and, compared with the lowest quartile, the risk of T2DM for the highest quartile was reduced (OR $=0.40,95 \%$ CI: 0.25, 0.63, $\left.P_{\text {for trend }}<0.001\right)$. There was no significant association between the risk of T2DM and the 
Table 3 OR of T2DM for quartiles of six main food groups intake in Guangzhou, China

\begin{tabular}{|c|c|c|c|c|c|c|c|c|}
\hline \multirow[b]{3}{*}{ Food group } & \multicolumn{7}{|c|}{ Quartiles of dietary energy-adjusted intake } & \multirow[b]{3}{*}{$P_{\text {for trend }}$} \\
\hline & \multirow[b]{2}{*}{ Q1 (referent) } & \multicolumn{2}{|c|}{ Q2 } & \multicolumn{2}{|c|}{ Q3 } & \multicolumn{2}{|c|}{ Q4 (highest) } & \\
\hline & & OR & $95 \% \mathrm{Cl}$ & OR & $95 \% \mathrm{Cl}$ & OR & $95 \% \mathrm{Cl}$ & \\
\hline \multicolumn{9}{|l|}{ Total grains } \\
\hline *Intake (male/female), g/d & $202 / 148$ & $266 / 186$ & & $312 / 214$ & & $354 / 264$ & & \\
\hline$n$ (case-control) & 141/192 & 69/192 & & 67/192 & & 107/192 & & \\
\hline ORI† & 1.00 & 0.50 & $0.35,0.71$ & 0.50 & $0.35,0.71$ & 0.78 & $0.56,1.08$ & 0.062 \\
\hline ORII & 1.00 & 0.64 & $0.41,0.99$ & 0.55 & $0.35,0.84$ & 0.73 & $0.48,1.12$ & 0.081 \\
\hline \multicolumn{9}{|l|}{ Fruits } \\
\hline *Intake (male/female), $\mathrm{g} / \mathrm{d}$ & $33 / 38$ & $87 / 99$ & & $146 / 156$ & & $256 / 268$ & & \\
\hline$n$ (case-control) & $165 / 192$ & $83 / 192$ & & $66 / 192$ & & 70/192 & & \\
\hline ORI† & 1.00 & 0.49 & $0.36,0.68$ & 0.40 & $0.28,0.57$ & 0.41 & $0.28,0.58$ & $<0.001$ \\
\hline ORII & 1.00 & 0.49 & $0.32,0.75$ & 0.41 & $0.26,0.63$ & 0.46 & $0.29,0.72$ & $<0.001$ \\
\hline \multicolumn{9}{|l|}{ Vegetables } \\
\hline *Intake (male/female), g/d & $196 / 188$ & $291 / 285$ & & $388 / 385$ & & $538 / 537$ & & \\
\hline$n$ (case-control) & $88 / 192$ & 95/192 & & 99/192 & & 102/192 & & \\
\hline ORI† & 1.00 & 1.07 & $0.76,1.52$ & $1 \cdot 12$ & $0.80,1.58$ & $1 \cdot 16$ & $0.82,1.64$ & 0.389 \\
\hline ORII & 1.00 & 1.44 & $0.92,2.26$ & 1.27 & $0.83,1.94$ & 1.38 & $0.90,2 \cdot 12$ & 0.222 \\
\hline \multicolumn{9}{|l|}{ Animal foods } \\
\hline *Intake (male/female), g/d & $155 / 122$ & $227 / 173$ & & $284 / 219$ & & $386 / 301$ & & \\
\hline$n$ (case-control) & 109/192 & 79/192 & & $81 / 192$ & & $115 / 192$ & & \\
\hline ORI† & 1.00 & 0.73 & $0.51,1.03$ & 0.76 & $0.54,1.07$ & 1.06 & $0.76,1.46$ & 0.715 \\
\hline ORII & 1.00 & 0.87 & $0.57,1.33$ & 1.00 & $0.66,1.51$ & 1.08 & $0.72,1.63$ & 0.632 \\
\hline \multicolumn{9}{|l|}{ Milk } \\
\hline *Intake (male/female), g/d & $2 \cdot 2 / 4 \cdot 3$ & $21 \cdot 8 / 71 \cdot 8$ & & $83 \cdot 9 / 172 \cdot 6$ & & $266 \cdot 8 / 298 \cdot 0$ & & \\
\hline$n$ (case-control) & $125 / 192$ & 67/192 & & 95/192 & & 97/192 & & \\
\hline ORI† & 1.00 & 0.52 & $0.36,0.75$ & 0.76 & $0.54,1.05$ & 0.71 & $0.53,1.07$ & 0.292 \\
\hline ORII‡ & 1.00 & 0.52 & $0.33,0.84$ & 0.64 & $0.41,0.99$ & 0.51 & $0.33,0.81$ & 0.012 \\
\hline \multicolumn{9}{|l|}{ Cooking oil } \\
\hline *Intake (male/female), g/d & $8 \cdot 0 / 8 \cdot 3$ & $13 \cdot 7 / 11 \cdot 6$ & & $21 \cdot 5 / 17 \cdot 7$ & & $31 \cdot 9 / 28 \cdot 2$ & & \\
\hline$n$ (case-control) & 29/192 & $38 / 192$ & & $94 / 192$ & & 223/192 & & \\
\hline ORI† & 1.00 & $1 \cdot 20$ & $0.69,2.08$ & $3 \cdot 11$ & $1.93,5.00$ & $7 \cdot 82$ & $4 \cdot 97,12 \cdot 31$ & $<0.001$ \\
\hline ORII & 1.00 & 1.38 & $0.70,2.74$ & 4.04 & $2 \cdot 25,7 \cdot 24$ & $10 \cdot 85$ & $6 \cdot 14,19.17$ & $<0.001$ \\
\hline
\end{tabular}

T2DM, type 2 diabetes mellitus.

*Median intake in male/female controls at each quantile after energy-adjusted.

†No factor adjusted.

†Adjusted for age, sex, marital status, educational level, occupation, smoking, alcohol consumption, tea drinking, economic conditions, energy intake, physical activity and family history of T2DM.

high-light-coloured vegetable and low-grain dietary pattern and the high-dark-coloured vegetable dietary pattern.

\section{Discussion}

Various foods are associated with or protect against T2DM, but the dietary patterns are often population-specific. This study aimed to assess the associations between the foods and dietary patterns and the risk of T2DM in South China. The results suggest that in subjects from South China, a high-fruit dietary pattern is associated with a lower risk of T2DM.

In this study, conditional logistic regression analyses were performed to investigate the associations between the intake of different food categories and the risk of T2DM. In addition, the factor analysis method was used to construct possible dietary patterns, and the scores were used as the dietary evaluation method to explore the correlation between the overall quality of diet and the risk of T2DM. The results showed that a dietary pattern characterised by a moderate intake of grains and carbohydrates, high intake of whole grains, various fruits, milk, fresh beans and mixed dry beans could reduce the risk of T2DM. Among them, whole grains, fruits and milk had more significant protective effects, and the higher the intake, the stronger the protective effect. Refined grains with more cooking oils and carbohydrates, high meat intake, and low grain intake may increase the likelihood of developing T2DM, which needs further confirmation. In fact, the protective dietary pattern described above (whole grains, various fruits, milk, fresh beans, mixed beans and high fruit) is similar to the Mediterranean dietary pattern (olive oil, cereals, fruits, vegetables, nuts, seeds, fish, seafood and dairy) ${ }^{(27)}$, which is already well known to be associated with a decreased risk of $\mathrm{T}_{2} \mathrm{DM}^{(28)}$. On the other hand, the dietary pattern observed here as being associated with an increased risk of T2DM (refined grains with more cooking oils and carbohydrates, high meat intake, and low grain intake) is similar to the Western dietary pattern (fried foods, refined grains, and processed and red meat $)^{(29)}$, which is already well known to be associated with the epidemics of obesity, diabetes and metabolic syndrome in Western countries ${ }^{(30)}$. Supporting these results, a recent Chinese study showed 
Table 4 OR of T2DM for quartiles by subgroups of vegetables and fruits in Guangzhou, China

\begin{tabular}{|c|c|c|c|c|c|c|c|c|}
\hline \multirow[b]{3}{*}{ Food group } & \multicolumn{7}{|c|}{ Quartiles of dietary energy-adjusted intake } & \multirow[b]{3}{*}{$P_{\text {for trend }}$} \\
\hline & \multirow[b]{2}{*}{ Q1 (referent) } & \multicolumn{2}{|c|}{ Q2 } & \multicolumn{2}{|c|}{ Q3 } & \multicolumn{2}{|c|}{ Q4 (highest) } & \\
\hline & & OR & $95 \% \mathrm{Cl}$ & OR & $95 \% \mathrm{Cl}$ & OR & $95 \% \mathrm{Cl}$ & \\
\hline \multicolumn{9}{|l|}{ Dark-coloured vegetables } \\
\hline Intake* (male/female), g/d & $138 / 124$ & 208/199 & & $285 / 275$ & & $419 / 400$ & & \\
\hline$n$ (case-control) & 93/192 & $91 / 192$ & & 100/192 & & 100/192 & & \\
\hline ORI† & 1.00 & 0.98 & $0.69,1.38$ & 1.07 & $0.76,1.51$ & 1.08 & $0.76,1.53$ & 0.561 \\
\hline ORII & 1.00 & 0.89 & $0.57,1.38$ & $1 \cdot 18$ & $0.77,1.83$ & 1.47 & $0.97,2.23$ & 0.084 \\
\hline \multicolumn{9}{|l|}{ Light-coloured vegetables } \\
\hline Intake* (male/female), g/d & $44 / 48$ & $101 / 97$ & & $165 / 159$ & & $289 / 279$ & & \\
\hline$n$ (case-control) & 108/192 & $111 / 192$ & & 79/192 & & 86/192 & & \\
\hline ORI† & 1.00 & 1.03 & $0.74,1.43$ & 0.73 & $0.52,1.04$ & 0.80 & $0.56,1.13$ & 0.069 \\
\hline ORII & 1.00 & 1.47 & $0.95,2.27$ & 1.29 & $0.83,2.00$ & 1.33 & $0 \cdot 84,2 \cdot 11$ & 0.364 \\
\hline \multicolumn{9}{|l|}{ Root vegetables } \\
\hline Intake* (male/female), g/d & $10 / 9$ & $27 / 26$ & & $50 / 46$ & & $101 / 95$ & & \\
\hline$n$ (case-control) & $159 / 192$ & 84/192 & & 76/192 & & 65/192 & & \\
\hline ORI† & 1.00 & 0.72 & $0.52,1.00$ & 0.66 & $0.48,0.91$ & 0.66 & $0.46,0.93$ & 0.007 \\
\hline ORII & 1.00 & 0.96 & $0.65,1.44$ & 0.80 & $0.54,1.19$ & 0.76 & $0.49,1.18$ & 0.099 \\
\hline \multicolumn{9}{|l|}{ Dark-coloured fruits } \\
\hline Intake* (male/female), g/d & $7 / 9$ & $21 / 25$ & & $42 / 46$ & & $105 / 100$ & & \\
\hline$n$ (case-control) & $136 / 192$ & 93/192 & & 80/192 & & 75/192 & & \\
\hline ORI† & 1.00 & 0.68 & $0.48,0.96$ & 0.58 & $0.42,0.83$ & 0.54 & $0.38,0.77$ & $<0.001$ \\
\hline ORII & 1.00 & 0.71 & $0.47,1.08$ & 0.50 & $0.33,0.77$ & 0.58 & $0.37,0.90$ & 0.003 \\
\hline \multicolumn{9}{|l|}{ Light-coloured fruits } \\
\hline Intake* (male/female), g/d & $20 / 19$ & $54 / 53$ & & $96 / 94$ & & $175 / 178$ & & \\
\hline$n$ (case-control) & $137 / 192$ & $91 / 192$ & & 76/192 & & 80/192 & & \\
\hline ORI† & 1.00 & 0.52 & $0.31,0.85$ & 0.39 & $0.24,0.64$ & 0.37 & $0.22,0.61$ & $<0.001$ \\
\hline ORII & 1.00 & 0.45 & $0.29,0.70$ & 0.40 & $0.26,0.63$ & 0.39 & $0.25,0.60$ & 0.003 \\
\hline
\end{tabular}

T2DM, type 2 diabetes mellitus.

*Median intake in male/female controls at each quantile after energy-adjusted.

†No factor adjusted.

¥Adjusted for age, sex, marital status, educational level, occupation, smoking, alcohol consumption, tea drinking, economic conditions, energy intake, physical activity and family history of T2DM.

Table 5 OR of T2DM for quartiles of dietary patterns in Guangzhou, China

\begin{tabular}{|c|c|c|c|c|c|c|c|c|}
\hline \multirow[b]{3}{*}{ Dietary pattern } & \multicolumn{7}{|c|}{ Quartiles of dietary energy-adjusted intake } & \multirow[b]{3}{*}{$P_{\text {for trenc }}$} \\
\hline & \multirow[b]{2}{*}{ Q1 (Referent) } & \multicolumn{2}{|c|}{ Q2 } & \multicolumn{2}{|c|}{ Q3 } & \multicolumn{2}{|c|}{ Q4 (Highest) } & \\
\hline & & OR & $95 \% \mathrm{Cl}$ & OR & $95 \% \mathrm{Cl}$ & OR & $95 \% \mathrm{Cl}$ & \\
\hline \multicolumn{9}{|c|}{ High-light-coloured vegetable and low-grain } \\
\hline$n$ (case-control) & $93 / 192$ & 94/192 & & $97 / 192$ & & $100 / 192$ & & \\
\hline ORI ${ }^{*}$ & 1.00 & 0.96 & $0.66,1.40$ & 1.07 & $0.73,1.56$ & 1.02 & $0.70,1.47$ & 0.801 \\
\hline ORII† & 1.00 & 1.09 & $0.69,1.73$ & 1.43 & $0.90,2.27$ & 1.22 & $0.76,1.96$ & 0.259 \\
\hline ORIII‡ & 1.00 & $1 \cdot 24$ & $0.77,1.99$ & 1.67 & $1.03,2.69$ & 1.59 & $0.96,2.63$ & 0.860 \\
\hline \multicolumn{9}{|l|}{ High-fruit } \\
\hline$n$ (case-control) & $143 / 192$ & $149 / 192$ & & $88 / 192$ & & 67/192 & & \\
\hline ORI ${ }^{*}$ & 1.00 & 0.53 & $0.37,0.76$ & 0.48 & $0.33,0.69$ & 0.39 & $0.27,0.57$ & $<0.001$ \\
\hline ORII & 1.00 & 0.49 & $0.32,0.77$ & 0.50 & $0.32,0.79$ & 0.42 & $0.26,0.68$ & $<0.001$ \\
\hline ORIII‡ & 1.00 & 0.46 & $0.30,0.71$ & 0.48 & $0.31,0.74$ & 0.40 & $0.25,0.63$ & $<0.001$ \\
\hline \multicolumn{9}{|c|}{ High-red meat and low-grain } \\
\hline$n$ (case-control) & $91 / 192$ & 87/192 & & $78 / 192$ & & $128 / 192$ & & \\
\hline ORI ${ }^{*}$ & 1.00 & 0.96 & $0.66,1.39$ & $0 \cdot 87$ & $0.59,1.27$ & 1.46 & $1.02,2.09$ & 0.053 \\
\hline ORII† & 1.00 & 0.88 & $0.56,1.39$ & 0.93 & $0.59,1.46$ & 1.53 & $1.00,2.35$ & 0.056 \\
\hline ORIII‡ & 1.00 & 0.89 & $0.56,1.40$ & 0.93 & $0.59,1.47$ & 1.50 & $0.97,2.32$ & 0.072 \\
\hline \multicolumn{9}{|c|}{ High-dark-coloured vegetable } \\
\hline$n$ (case-control) & $91 / 192$ & 78/192 & & $96 / 192$ & & 119/192 & & \\
\hline ORI ${ }^{*}$ & 1.00 & 0.82 & $0.55,1.21$ & 0.97 & $0.68,1.40$ & $1 \cdot 32$ & $0.92,1.89$ & 0.078 \\
\hline ORII† & 1.00 & 0.89 & $0.55,1.43$ & 1.00 & $0.64,1.56$ & 1.52 & $0.98,2.35$ & 0.075 \\
\hline ORIII‡ & 1.00 & 1.03 & $0.63,1.69$ & 1.33 & $0.82,2 \cdot 13$ & $2 \cdot 72$ & $1.63,4.54$ & 0.074 \\
\hline
\end{tabular}

T2DM, type 2 diabetes mellitus.

${ }^{*}$ No factor adjusted.

†Adjusted for age, sex, marital status, educational level, occupation, smoking, alcohol consumption, tea drinking, economic conditions, energy intake, physical activity and family history of T2DM.

$\ddagger$ Adjusted for the covariates in model 2 plus dietary fibre. 
that dietary patterns rich in refined grains increased the risk of T2DM, while those rich in whole grains decreased the risk of $\mathrm{T}_{2} \mathrm{DM}^{(31)}$. A study from Zhejiang Province (East China) showed that the Western diet was associated with an increased risk of T2DM, while a dietary pattern characterised by grains and vegetables was associated with a lower risk ${ }^{(32)}$. The Singapore Health Study revealed that a dietary pattern characterised by vegetables, fruits and soya foods was beneficial against T2DM, while a high intake of dim sum, meat, sweetened food/beverages and fried foods was associated with a high risk of $\mathrm{T} 2 \mathrm{DM}^{(33)}$. Those results are supported by two meta-analyses that showed that whole grains, fruits and milk products decreased the risk of T2DM, while red meat, processed meat and sugar-sweetened beverages increased this risk $^{(34,35)}$. A combined analysis of the Nurses' Health Study, Nurses' Health Study 2 and Health Professionals' Follow-Up Study also supports those findings, that is, that high intake of coffee, whole grains, fruits and nuts is associated with a decreased risk of T2DM, while a high intake of meats, refined grains and sweetened beverages increased the risk ${ }^{(36)}$. Similar, but not identical, dietary patterns have been observed by other studies ${ }^{(5,21,35)}$. Nevertheless, caution must be taken when comparing dietary patterns from all around the globe because of the cultural differences in diet that cannot be taken into account by the food questionnaires.

There is increasing evidence that the intake of whole grains in substitution to refined grains reduces the risk of $\mathrm{T}_{2} \mathrm{DM}^{(37)}$. Whole grains have a high content of dietary fibres that contribute to decreasing the energy density and higher gastric distention and to increase hormones associated with energy and glucose homoeostasis ${ }^{(38)}$. The structural integrity of whole grains requires a higher chewing rate, leading to earlier satiety that benefits body weight control ${ }^{(39)}$. A meta-regression analysis showed that consuming $45 \mathrm{~g} / \mathrm{d}$ of whole grains would reduce the T2DM risk by $20 \%$ compared with $7.5 \mathrm{~g} / \mathrm{d}^{(40)}$. In the present study, patients with T2DM consumed less whole-grain foods compared with controls, but the amount was relatively low in both groups.

The results of previous studies regarding vegetable and fruit intakes and the risk of T2DM are inconsistent, but the majority reported protective effects. Although Harding et al. $^{(16)}$ showed that the association between fruit and vegetable intake with T2DM was only significant for the lowest quintile, a meta-analysis by Li et al. ${ }^{(41)}$ showed that a high intake of fruits or green leafy vegetables was associated with a markedly lower risk of T2DM. Shu et al. ${ }^{(32)}$ showed that vegetables, not fruits, were associated with a decreased risk of T2DM, while Odegaard et al. ${ }^{(33)}$ showed that both vegetables and fruits were protective against T2DM. Many dark green vegetables and fruits (except bananas) have high levels of carotenoids but contain little or no starch, whereas avocado, maize and potatoes are full of starch, indicating the importance of dividing the vegetables or fruits into separate categories to avoid the influence of different nutrient content ${ }^{(38)}$. Consistent with the metaanalysis study by Wang PY et al. ${ }^{(42)}$, both of us have found null association between intake of total vegetables with T2DM, but there were inconsistent and insignificant associations between green leafy, yellow, cruciferous vegetables and diabetes in our study, and the different findings of total vegetables and specific vegetables strengthened the potential heterogeneity due to the difference in the vegetables categories of subgroup analyses between their study and ours. On the one hand, vegetables and fruits have been placed in dietary guidance due to their high concentrations of vitamins, antioxidants, minerals, phytochemicals and dietary fibres ${ }^{(38)}$. On the other hand, vegetables and fruits are good sources of $\mathrm{K}$, and their energy density is low; the mechanism in the prevention of T2DM could be similar to that of whole grains and beans ${ }^{(41)}$, but studies of fruit fibres on T2DM yielded inconsistent results ${ }^{(43)}$. Fruits are also rich in micronutrients, especially vitamins and antioxidants $^{(44)}$, which are well known to protect against the development of T2DM. The Mg contained in fruits is also inversely correlated with the risk of diabetes ${ }^{(45)}$. Fruits have a low energy density, contributing to weight loss ${ }^{(16)}$. In the present study, fruit intake is the strongest contributor to Pattern 2 against T2DM. This is supported by a Chinese longitudinal study that showed that fresh fruit intake protects against incident diabetes ${ }^{(46)}$.

The present study showed that beans and dairy were part of the dietary pattern for protection against T2DM, while meat (especially red meat intake) was associated with an increased risk of T2DM. Replacing white rice by beans has been shown to improve cardiometabolic indicators of $\mathrm{T} 2 \mathrm{DM}^{(47)}$. Beans also contain fibres, which act in the same way as fibres from whole grains. Dairy contains a wide variety of vitamins and minerals that could play roles in the prevention of $\mathrm{T} 2 \mathrm{DM}^{(48)}$, and bacteria contained in dairy products like yogurt and cheese also play roles in glucose homoeostasis $^{(49)}$. The present study found total milk intake was associated with a low risk of T2DM, while Marangoni $\mathrm{F}$ et al $^{(50)}$ indicate that the protective association between milk and diabetes seems to apply to lowfat milk but not to full-fat milk. The inconsistent results might be due to the low consumption of total milk among Chinese populations, especially low intake of low-fat milk and skim milk ${ }^{(51)}$. Red meat and processed meat have been reported to have a positive correlation with T2DM in a number of studies, and processed meats are also known to be unhealthy ${ }^{(21,34,35,52)}$. Although the exact mechanisms through which meats increase the risk of T2DM is unknown, it is known that body weight can be affected by specific amino acids found in meat, as well as by SFA ${ }^{(52)}$.

A dietary pattern characterised by whole grains, various fruits, milk, fresh beans, mixed beans and high fruit has been suggested to be used in the management of T2DM. Indeed, a Chinese longitudinal study showed that fresh fruit intake protects against vascular complications of $\mathrm{T} 2 \mathrm{DM}^{(46)}$. 
A high intake of fruits is also recommended by the dietary guidelines for $\mathrm{T}_{2} \mathrm{DM}^{(53)}$. Furthermore, the Mediterranean diet not only protects against T2DM but it is also suggested as part of the management of $\mathrm{T} 2 \mathrm{DM}^{(27)}$. Actually, the Mediterranean diet has been shown to improve glycemic control in $\mathrm{T}_{2} \mathrm{DM}^{(54)}$. This shows the importance of consuming healthy foods, which should decrease the mortality and morbidity of T2DM, saving lives and financial resources.

This study has some strengths. This was a T2DM casecontrol study with strictly implemented inclusion and exclusion criteria. New-onset diabetic patients (those diagnosed within 3 months of enrolment) were included in the study, and those with recently measured normal values of FBG were included as controls. This measure improved the representativeness of cases and prevented changes in dietary habits due to T2DM. For the T2DM and control groups, patients with diseases or conditions that may affect outcomes (such as insulin resistance) were excluded, thus minimising the potential confounding factors. The FFQ that we used has shown good reliability and validity for dietary assessment, as validated in a local female population ${ }^{(23)}$. Finally, the combination of a single nutrient, food group and dietary pattern was used to explore and verify factors that may protect against T2DM in the diet, which was also beneficial to the direct promotion and application of this research findings.

This study also has some limitations. Given it was a case-control study, there may have selection bias and unclear causal relationships, but we tried to reduce this bias by recruiting the case patients and control subjects from a variety of sources (two hospitals and one community). A FFQ was used to investigate the dietary intake of new-onset diabetic patients and control subjects over the past year, and recall bias is inevitable ${ }^{(55)}$, but this recall error is unlikely to differ between the patients and control subjects. Moreover, there may be a selection bias in selfreported information by the patients with T2DM, since patients tend to change their dietary habits after diseases, but adults generally maintain a long-term stable dietary habit ${ }^{(56)}$, and we included only incident cases and meticulously excluded participants with a history of other chronic diseases that might change dietary habits or nutritional factors, and our dietary intake assessment covering the year prior to diagnosis or interview was judged to reflect the participants' long-term dietary habits and was unlikely to produce an inverse causal relationship. Finally, the subjects of this study were all from the main geographical area (South China), which limits generalisability.

In conclusion, in Chinese subjects from Guangzhou, fruit intake has protective effects against T2DM. A high-fruit dietary pattern is associated with a lower risk of T2DM. Other dietary factors that protected against T2DM included whole grains, milk, fruits, total grains and an appropriate amount of vegetables. Large and well-designed studies are recommended to confirm this conclusion.

\section{Acknowledgements}

Acknowledgements: We thank all the people who voluntarily participated in the study and the health care workers in the community and hospitals for recruiting the subjects. We thank Yuanting He in assisting the questionnaire survey. Financial support: This work was supported by the Youth Fund Project of the National Natural Science Foundation of China, grant number 81202197. Conflict of interest: None. Authorship: YY and YC conceived and designed the study. SZ, WL and YY contributed to data collection. HH, YS, SW and PC conducted data cleaning and analysis, overseen by YL and SF. YY wrote the original main manuscript. $\mathrm{YY}$ and $\mathrm{SZ}$ produced the final revised manuscript. YC and YY reviewed the paper and approved its final version. All authors were involved in interpreting results, editing the manuscript for contents and approved to the final version to be published. Ethics of human subject participation: This study was conducted according to the guidelines laid down in the Declaration of Helsinki and all procedures involving human subjects were approved by the ICE for Clinical Research and Animal Trials of the First Affiliated Hospital of Sun Yat-sen University. Written informed consent was obtained from all subjects.

\section{Supplementary material}

For supplementary material accompanying this paper visit https://doi.org/10.1017/S1368980020004930

\section{References}

1. (2019) Introduction: standards of medical care in diabetes2019. Diabetes care 42, S1-S2.

2. Pan XR, Yang WY, Li GW et al. (1997) Prevalence of diabetes and its risk factors in China, 1994. National Diabetes Prevention and Control Cooperative Group. Diabetes care 20, 1664-1669.

3. Wang L, Gao P, Zhang M et al. (2017) Prevalence and ethnic pattern of diabetes and Prediabetes in China in 2013. JAMA 317, 2515-2523.

4. Hu H, Sawhney M, Shi L et al. (2015) A systematic review of the direct economic burden of type 2 diabetes in china. Diabetes Ther 6, 7-16.

5. Sami W, Ansari T, Butt NS et al. (2017) Effect of diet on type 2 diabetes mellitus: a review. Int J Health Sci 11, 65-71.

6. Shen X, Vaidya A, Wu S et al. (2016) The Diabetes epidemic in China: an integrated review of national surveys. Endocr Pract 22, 1119-1129.

7. Micha R, Wallace SK \& Mozaffarian D (2010) Red and processed meat consumption and risk of incident coronary heart disease, stroke, and diabetes mellitus: a systematic review and meta-analysis. Circulation 121, 2271-2283.

8. Pan A, Sun Q, Bernstein AM et al. (2013) Changes in red meat consumption and subsequent risk of type 2 diabetes mellitus: three cohorts of US men and women. JAMA Intern Med 173, 1328-1335.

9. Cahill LE, Pan A, Chiuve SE et al. (2014) Fried-food consumption and risk of type 2 diabetes and coronary artery disease: a prospective study in 2 cohorts of US women and men. Am J Clin Nutr 100, 667-675. 
10. Hu EA, Pan A, Malik V et al. (2012) White rice consumption and risk of type 2 diabetes: meta-analysis and systematic review. BMJ 344, e1454.

11. Imamura F, O'Connor L, Ye Z et al. (2015) Consumption of sugar sweetened beverages, artificially sweetened beverages, and fruit juice and incidence of type 2 diabetes: systematic review, meta-analysis, and estimation of population attributable fraction. BMJ 351, h3576.

12. Villegas R, Liu S, Gao YT et al. (2007) Prospective study of dietary carbohydrates, glycemic index, glycemic load, and incidence of type 2 diabetes mellitus in middle-aged Chinese women. Arch Intern Med 167, 2310-2316.

13. Deng A, Pattanaik S, Bhattacharya A et al. (2018) Fish consumption is associated with a decreased risk of death among adults with diabetes: 18-year follow-up of a national cohort. Nutr Metab Cardiovasc Dis 28, 1012-1020.

14. Alvarez-Bueno C, Cavero-Redondo I, Martinez-Vizcaino V et al. (2019) Effects of milk and dairy product consumption on type 2 diabetes: overview of systematic reviews and metaanalyses. Adv Nutr 10, S154-S163.

15. Mueller NT, Odegaard AO, Gross MD et al. (2012) Soy intake and risk of type 2 diabetes in Chinese Singaporeans [corrected]. Eur J Nutr 51, 1033-1040.

16. Harding AH, Wareham NJ, Bingham SA et al. (2008) Plasma vitamin $C$ level, fruit and vegetable consumption, and the risk of new-onset type 2 diabetes mellitus: the European prospective investigation of cancer-Norfolk prospective study. Arch Intern Med 168, 1493-1499.

17. Hooson Jzh J, Hutchinson Jyh J, Warthon-Medina $\mathrm{M}$ et al. (2019) A systematic review of reviews identifying UK validated dietary assessment tools for inclusion on an interactive guided website for researchers: www.nutritools.org. Crit Rev Food Sci Nutr 60, 1-25.

18. Ma G (2015) Food, eating behavior, and culture in Chinese society. J Ethnic Foods 2, 195-199.

19. Das SK \& Sharma NK (2014) Expression quantitative trait analyses to identify causal genetic variants for type 2 diabetes susceptibility. World J Diabetes 5, 97-114.

20. Prasad RB \& Groop L (2015) Genetics of type 2 diabetespitfalls and possibilities. Genes (Basel) 6, 87-123.

21. Jannasch F, Kroger J \& Schulze MB (2017) Dietary patterns and type 2 diabetes: a systematic literature review and meta-analysis of prospective studies. J Nutr 147, 1174-1182.

22. American Diabetes A (2007) Diagnosis and classification of diabetes mellitus. Diabetes care 30, S42-\$47.

23. Zhang CX \& Ho SC (2009) Validity and reproducibility of a food frequency questionnaire among Chinese women in Guangdong province. Asia Pac J Clin Nutr 18, 240-250.

24. Yang YX, Wang GY \& Pan XC (2002) China Food Composition Table. Beijing: Peking University Medical Press.

25. Wahid A, Manek N, Nichols M et al. (2016) Quantifying the association between physical activity and cardiovascular disease and diabetes: a systematic review and meta-analysis. J Am Heart Assoc 5, e002495.

26. Willett WC, Howe GR \& Kushi LH (1997) Adjustment for total energy intake in epidemiologic studies. Am J Clin Nutr 65 1220S-1228S.

27. Lacatusu CM, Grigorescu ED, Floria M et al. (2019) The mediterranean diet: from an environment-driven food culture to an emerging medical prescription. Int J Environ Res Public Health 16, 942.

28. Georgoulis M, Kontogianni MD \& Yiannakouris N (2014) Mediterranean diet and diabetes: prevention and treatment. Nutrients 6, 1406-1423.

29. Cordain L, Eaton SB, Sebastian A et al. (2005) Origins and evolution of the Western diet: health implications for the 21st century. Am J Clin Nutr 81, 341-354.

30. Adeva-Andany MM, Ranal-Muino E, Vila-Altesor $\mathrm{M}$ et al. (2019) Dietary habits contribute to define the risk of type 2 diabetes in humans. Clin Nutr ESPEN 34, 8-17.
31. Zhou J, Sheng J, Fan Y et al. (2019) Dietary patterns, dietary intakes and the risk of type 2 diabetes: results from the Hefei Nutrition and Health Study. Int J Food Sci Nutr 70, 412-420.

32. Shu L, Shen XM, Li C et al. (2017) Dietary patterns are associated with type 2 diabetes mellitus among middle-aged adults in Zhejiang Province, China. Nutr J 16, 81.

33. Odegaard AO, Koh WP, Butler LM et al. (2011) Dietary patterns and incident type 2 diabetes in chinese men and women: the Singapore Chinese health study. Diabetes care 34, 880-885.

34. Schwingshackl L, Hoffmann G, Lampousi AM et al. (2017) Food groups and risk of type 2 diabetes mellitus: a systematic review and meta-analysis of prospective studies. Eur $J$ Epidemiol 32, 363-375.

35. Alhazmi A, Stojanovski E, McEvoy M et al. (2014) The association between dietary patterns and type 2 diabetes: a systematic review and meta-analysis of cohort studies. $J$ Hum Nutr Diet 27, 251-260.

36. Ardisson Korat AV, Willett WC \& Hu FB (2014) Diet, lifestyle, and genetic risk factors for type 2 diabetes: a review from the nurses' Health Study, Nurses' Health Study 2, and Health Professionals' follow-up study. Curr Nutr Rep 3, 345-354.

37. Della Pepa G, Vetrani C, Vitale M et al. (2018) Wholegrain intake and risk of type 2 diabetes: evidence from epidemiological and intervention studies. Nutrients 10, 1288.

38. Sanchez D, Miguel M \& Aleixandre A (2012) Dietary fiber, gut peptides, and adipocytokines. J Med Food 15, 223-230.

39. Wanders AJ, van den Borne JJ, de Graaf C et al. (2011) Effects of dietary fibre on subjective appetite, energy intake and body weight: a systematic review of randomized controlled trials. Obes Rev 12, 724-739.

40. Chanson-Rolle A, Meynier A, Aubin F et al. (2015) Systematic review and meta-analysis of human studies to support a quantitative recommendation for whole grain intake in relation to type 2 diabetes. PLoS One 10, e 0131377.

41. Li M, Fan Y, Zhang X et al. (2014) Fruit and vegetable intake and risk of type 2 diabetes mellitus: meta-analysis of prospective cohort studies. BMJ Open 4, e005497.

42. Wang PY, Fang JC, Gao ZH et al. (2016) Higher intake of fruits, vegetables or their fiber reduces the risk of type 2 diabetes: a meta-analysis. J Diabetes Investig 7, 56-69.

43. Schulze MB, Schulz M, Heidemann C et al. (2007) Fiber and magnesium intake and incidence of type 2 diabetes: a prospective study and meta-analysis. Arch Intern Med 167, 956-965.

44. Hozawa A, Jacobs DR, Steffes MW et al. (2006) Associations of serum carotenoid concentrations with the development of diabetes and with insulin concentration: interaction with smoking: the Coronary Artery Risk Development in Young Adults (CARDIA) study. Am J Epidemiol 163, 929-937.

45. Larsson SC \& Wolk A (2007) Magnesium intake and risk of type 2 diabetes: a meta-analysis. I Intern Med 262, 208-214.

46. Du H, Li L, Bennett D et al. (2017) Fresh fruit consumption in relation to incident diabetes and diabetic vascular complications: a $7-y$ prospective study of 0.5 million Chinese adults. PLoS Med 14, e1002279.

47. Mattei J, Malik V, Wedick NM et al. (2015) Reducing the global burden of type 2 diabetes by improving the quality of staple foods: the Global Nutrition and Epidemiologic Transition Initiative. Glob Health 11, 23.

48. Guo J, Givens DI, Astrup A et al. (2019) The Impact of dairy products in the development of type 2 diabetes: where does the evidence stand in 2019? Adv Nutr 10, 1066-1075.

49. Tonucci LB, Olbrich Dos Santos KM, Licursi de Oliveira L et al. (2017) Clinical application of probiotics in type 2 diabetes mellitus: a randomized, double-blind, placebocontrolled study. Clin Nutr 36, 85-92.

50. Marangoni F, Pellegrino L, Verduci E et al. (2019) Cow's milk consumption and health: a health professional's guide. $J \mathrm{Am}$ Coll Nutr 38, 197-208. 
51. Su C, Zhang B, Wang H et al. (2018) Milk consumption and effects on dietary calcium among Chinese aged 45 and above in 15 provinces, 2015. Wei Sheng Yan Jiu 47, 194-198.

52. Mari-Sanchis A, Gea A, Basterra-Gortari FJ et al. (2016) Meat consumption and risk of developing type 2 diabetes in the SUN Project: a highly educated middle-class population. PLoS One 11, e0157990.

53. Forouhi NG, Misra A, Mohan V et al. (2018) Dietary and nutritional approaches for prevention and management of type 2 diabetes. BMJ 361, k2234.
54. Papamichou D, Panagiotakos DB \& Itsiopoulos C (2019) Dietary patterns and management of type 2 diabetes: a systematic review of randomised clinical trials. Nutr Metab Cardiovasc Dis 29, 531-543.

55. Shim JS, Oh K \& Kim HC (2014) Dietary assessment methods in epidemiologic studies. Epidemiol Health 36, e2014009.

56. Macdonald HM, New SA \& Reid DM (2005) Longitudinal changes in dietary intake in Scottish women around the menopause: changes in dietary pattern result in minor changes in nutrient intake. Public Health Nutr 8, 409-416. 